\title{
Biomedical Education and Research: Expectations and Limitations
}

\author{
Gundu HR Rao* \\ Laboratory Medicine and Pathology, University of Minnesota, USA
}

Received: 制: September 19, 2018; Published: 畊 September 26, 2018

*Corresponding author: Gundu HR Rao, Emeritus Professor, Laboratory Medicine and Pathology, Director, Thrombosis Research, Lillehei Heart Institute, University of Minnesota, 12500 Park Potomac Ave, Unit 306N, Potomac MD, 20854, USA

\begin{abstract}
The Molecules in Medical Sciences is a course that is offered, to the first year Medical Students at the Department of Biochemistry, Cambridge University, UK, which focuses on diseases, which will be familiar by name and of high relevance, -like diabetes and cancer. Harvard Medical School on the other hand, says," preparation of medical school in the $21^{\text {st }}$ century, should reflect contemporary developments in medical knowledge, the pace of discovery, and the permeation of biochemistry, cell biology, and genetics into most areas of medicine". Oxford Royale Academy looks at biomedicine the following way, "Biochemistry, as the name suggests, is where Biology meets Chemistry: it is the study of the living things, at a molecular levelor, to put it another way, the study of the very foundations of life. At the Stanford University, the department of Biochemistry, founded by Arthur Kornberg, the Nobel Laureate, who received this award for his work on, "the mechanisms in the biological synthesis of deoxyribonucleic acid", focused for several decades, on DNA and RNA biochemistry.

The current approach is enormously diverse, with nearly everyone using interdisciplinary approaches of biochemistry, genetics, biophysics, structural biology, high resolution light microscopy, and other innovative methodologies. In addition to the training in human anatomy and physiology, present day medical students, also receive instruction in biochemistry, cell biology, embryology, nutrition, pathology, immunology, microbiology, molecular biology, neurobiology, nutrition, pathology, pharmacology, and virology. These foundational or basic sciences enable the future physician to understand, what constitutes the homeostasis of the healthy individual, the mechanisms by which that homeostasis is disrupted by disease, and how particular disease states may best be treated. New discoveries and advances, in these foundational sciences are so rapid, Harvard Medical School revises its curriculum fairly often. In a good well-rounded medical education, foundational sciences and clinical sciences are interdependent. Modern day biochemistry in the broadest sense, should provide needed insight into the underlying mechanisms of both structure and regulation, that occur at the molecular, cellular, tissue, organ, and whole system level. In this overview, we will try and attempt, to use this broadest definition, to describe the origin, progression, risk development, and precipitation of acute events leading to death. We will also discuss expectations and limitations, as we try to sketch chain of events, in the development of metabolic risks, progression of metabolic diseases, and the precipitation of ultimate acute event.
\end{abstract}

\section{Biomedical Education}

I have been debating for quite some time, as to how to improve teaching biochemistry to medical students. One may ask, as to why I should bother with this topic? It is more personal, than one can visualize. I graduated with my PhD degree from the department of Entomology, Kansas State University, specializing in Grain Science Technology. After my post-doctoral fellowship, I joined Dr. James G White, a pioneering platelet biologist, at the University of Minnesota, to help set up a biochemistry laboratory, to conduct biochemical studies which complement his studies on ultrastructure physiology and pathology. Professor James G White and I, shared NIH grants for over 30 years. As a director of Thrombosis Research, at the Lillehei Heart Institute, University of Minnesota, I worked on ultrastructure pathophysiology, biochemistry, and pharmacology of platelets, for four decades. Dr White and I, together have published over 1000 articles, which are reproduced in the book on platelets, that I am dedicating to the memory of Late James White my mentor [1].
My life and work at the University of Minnesota Medical School taught me the importance of multidisciplinary education and integrated approach to better healthcare. It is my sincere belief, that if I with Entomology background can develop a multidisciplinary knowledge of translational sciences, modern day students with well-rounded curricula can excel in both fundamental science, clinical sciences, and research. During the early part of my research training, I spent some time in the Biochemistry department of Royal College of Surgeons, Nuffield College, London. The Nuffield Foundation is a trust that has done much to stimulate new ideas in education in the UK. It is most famous for introducing, new curricula, for teaching science at all levels. Nuffield Foundation invited a group of distinguished people, to meet together in London in 1974, to discuss ways and means, to improve biochemistry teaching to medical students. The distinguished committee included professors (twenty-four) from various leading Universities of UK. 
The Chairman, Sir Charles Stuart-Harris, in his opening remarks said, that it was almost an invariable experience, when visiting medical schools, to hear complaints from the students, about the biochemistry teaching, that the subject was difficult, and that it had little relevance to the practice of medicine. Of course, the distinguished committee who met to discuss this important topic, were not able to provide all the answers, for this question, - how to make biochemistry more attractive to the medical students? In view of this concern, the committee recommended that biochemistry be taught in an interesting and relevant way, so that students have a framework on which, their future knowledge can be structured [2]. I mentioned earlier that at the Stanford University, Biochemistry department founded by Dr. Kornberg, major focus for decades was on DNA and RNA. Following the foot-steps of an accomplished innovator, was true at many other educational institutions. In a recent issue of NEJM, Francis Collins, the director of National Institutes of Health, USA, writes, that potential to alter human genes directly was first recognized nearly fifty years ago, at the same time advances in recombinant DNA technology were being made in the laboratories [3-5].

The story is the same in stem cell applications, as well as Regenerative medicine. Doris Taylor at the University of Minnesota developed "ghost hearts" from decellularized heart cell matrix using stem cells derived from humans and proclaimed that bioartificial heart was weeks away [4]. Translation of laboratory research to commercialization takes considerable time and in view of this fact, there exists a disconnect between the innovators, researchers, teachers, and clinicians. To bridge such knowledge gaps, half a century ago, at the University of Minnesota, the department of Laboratory Medicine and Pathology hired, dozens of basic science scientists, and offered courses in pathobiology, molecular biology, and mechanism of diseases. Some of these courses were abandoned for lack of sufficient response, from the medical students. How do we create interest in multidisciplinary studies? This will be a topic of my next overview. The extraordinary discoveries of the 20-th century medicine and technology, have created new challenges to biomedical education [6-11].

There is a great overall expectation, that the practice of medicine will change and introduce, precision medicine or personalized medicine. There is a recent emerging trend, with broader emphasis on using vast amounts of data, to serve "precision" ends, incorporating genomics as one of the many other associated fields of relevant technologies and innovations. In May of this year (2018), National Institutes of Health (NIH) USA, began enrollment of a vast medical research cohort. Named "All of US," it is meant to include 1 million U.S volunteers, who will be studied over 10 years at a cost of 1.45 billion. According an article in N. Engl. J. Med (2018. DOI:10.1056/NEJMp1806634), the project promises to "lay the scientific foundation for a new era of personalized, highly effective health care," a counterpart to previous "one size fits all" medicine." Having said that, the educators in majority of Institutions worldwide, are not ready to accept these changes and challenges. of course, incorporations of all the revolutionary innovations, will change the way the healthcare is delivered, but it requires the education of a new generation of translational, clinical scientists, physician scientists, and researchers as well as incorporation of these emerging technologies, in the biomedical education and research [6-12].

Now that I have briefly explained, what is happening with biomedical education and research, let me bring to the notice of the readers, what Harvard Medical School (MHS) is doing to accommodate these revolutionary changes. Beginning with the introduction in 2006 of a curriculum called, "New Integrated Curriculum" which emphasizes learning to learn, rather than rote memorization, and represents one of the most complete curricular reforms at a US Medical School. Students also are encouraged to complete a scholarly research project, after their clerkship-year. Dr. Robert Alpern, the dean of Yale Medical School said, "Harvard's curriculum change, is similar to a revision at Yale, and moving away from individual discipline-based courses to more integrated teaching, is where we need to go. Many schools are moving in similar direction". Now that I have reviewed briefly, the changes that are happening in the medical education, at least in the industrialized nations, I would like to discuss the advances in biomedical research that is taking place.

As the title of this essay indicates, I will describe what is known of the mechanisms, that underlie the pathogenesis of metabolic diseases, unmet expectations, reasons for the observed limitations. Future of medicine, especially the precision and personal medicine, lies in clinicians gaining much more detailed information about the patient, the underlying causes of the disease, the knowledge of the emerging technologies, and their applications, to deliver personalized or precision treatment, with a better outcome. Since my area of interest is cardiometabolic diseases, let me try to discuss how this is done at an academic setting.

\section{Biomedical Research}

Metabolic diseases such as hypertension, excess weight, obesity, endothelial dysfunction (arterial stiffness), subclinical atherosclerosis, metabolic syndrome, type-2 diabetes, and vascular diseases have reached epidemic proportions worldwide [13-17]. According to some expert's, cardiovascular disease has remained the biggest killer for over hundred years. At the University of Minnesota Rasmussen Center for Cardiovascular Disease Prevention, Professor Jay Cohn and associates have developed a philosophy, that focuses on treating the disease itself, rather managing just the risk factors [18]. How is it done? What really constitutes a metabolic disease? How can one develop clinical tests to find out the earliest alteration in the normal metabolic state? These are some important questions that come to my mind, when I think of efforts to reduce, reverse or prevent metabolic diseases. In a recent article in the Journal of American College of Cardiology, Professor Valentin Fuster and associates at the Mount Sinai Hospital, New York, reported their findings of a study, that closely looked at the clinical features of the participants from the Progression of Early Subclinical Atherosclerosis (PESA).

The specific objects of this study were, to seek to identify predictors of subclinical atherosclerosis, in cardiovascular risk- 
free individuals. They demonstrated, that after age and male sex, LDL- cholesterol was the main predictor for the presence of the atherosclerotic plaques, suggesting a great correlation between LDL-cholesterol and levels of atherosclerosis [19]. Using the latest non-invasive imaging technology, they demonstrated that plaques were present in $50 \%$ of the middle- aged individuals with no classical risk factors. Furthermore, these individuals also had hardened arteries showing the altered morphology and dysfunction of the vessel walls. They concluded, that, "these findings could help improve cardiovascular risk prevention in the general population, even before the appearance of conventional risk factors, an example of primoradial prevention". When developing strategies for the early diagnosis of complex and chronic diseases, such as hypertension, excess weight, obesity, type- 2 diabetes, and vascular diseases, it is important to discuss, how early is really early? In our opinion, the diagnosis and management of fetal origin of adult diseases would be the earliest? For instance, in the majority of Asian countries, even to this day, more than $30 \%$ of the children born are, low birth weight [20].

These low-birth-weight children, as they grow to adulthood, develop metabolic diseases [21]. The low-birth-weight (LBW) remains a high risk for infant mortality (NEJM 312:82-90, 1985). Collaborative Studies in India, from Medical Research Council (MRC) of United Kingdom (UK), with Mission Hospital Mysore (MHM), and King Edward Memorial (KEM) Hospital, Pune, have demonstrated that over a third of all babies born in India, are of low-birth-weight. Follow up of these LBW babies have demonstrated that they develop a significantly high incidence of elevated blood pressure, obesity, diabetes (type-2), metabolic syndrome, and cardiovascular diseases (CVDs). In view of these findings, MRC has set up an epidemiology resource center at KEM hospital Pune and at Mission Hospital, Mysore. This is not a finding that is unique to the South Asians. Xiao and associates in Beijing, China, studied individuals born between 1921 to 1954, at the Peking Union Medical College Hospital, to estimate the association between birth weight and Metabolic Syndrome [22].

In the 1980s, Barker developed a hypothesis according to which, many nutritional events that occur during the intrauterine growth, will influence the development of adult diseases [23-27]. In a way, it is a global problem, and needs immediate attention by the affected communities, as it seems to be one of the early causes for so called, "Fetal Origin of Adult Disease (FOAD)" [28]. There is extensive epidemiological evidence to support FOAD hypothesis. In brief, the hypothesis suggests, that intrauterine environmental exposures and events, affect the fetus development and thereby, increases the risk of specific diseases in adult life. Barker and colleagues documented initial support for this hypothesis for coronary artery disease (CAD). They standardized the mortality ratios for cardiovascular disease for 16,000 individuals, born in Hertfordshire, UK, from 1911-1930, to birth records for these individuals. The data suggested that low birth weight, small head circumference, and low mass/height index at birth, was associated with an increase in coronary heart disease in adulthood [28].
In view of the fact, that this probably is the earliest known risk for developing metabolic diseases, we have initiated a bilateral collaborative study between the researchers at the Children's National Medical Hospital (CNMH), Washington DC and the staff at the Diabetes Clinic, KEM Hospital, Pine, India, to explore the molecular mechanisms if any, that modulate these intrauterine alterations. Dr. Robert Freishtat and associates at the CNMH, Washington DC, have demonstrated, that visceral adipocytes shed exosomal-mediators (microRNAs), predicted to regulate key end-organ inflammatory and fibrotic pathways [29]. Preliminary studies between these two institutions, have been encouraging, and these studies have been funded by the prestigious National Institutes of Health (NIH) USA. Yet another molecular mechanism, that can predict the development of type- 2 diabetes is profiling of plasma free amino acids (PFAA). Several studies have consistently demonstrated, that increased levels of plasma and urinary branch chain amino acids (BCAAs), are associated with insulin resistance and have the quality to predict future diabetes development [30, 31].

How does this increase in the free amino acids occur? What metabolic alterations lead to this excess of essential free amino acids in the blood? Some studies are indicating that disruptions in gut microbiota composition could indeed change the amino acid metabolism. We have tens of trillion microbes in our gut, and they in turn have 3 million genes, capable of expressing proteins that can alter the metabolism of the host. The gut microbiota can be the source, for branched chain amino acids, as well as short-chain fatty acids. This could have significant implications, for developing insulin resistance as well as obesity. Talking about the role of gene expression and altered metabolism, NIH researchers link single gene variation to obesity. A single variation in the gene for brain-derived neurotropic factor (BDNF), may influence obesity in children and adults. In African American adults, the NIH team found that C-allele was associated with higher BMI and body fat percentage, in those with CT or CC types (NIH Public Release:29 oct 2015).

When in search of early markers of metabolic risks, the increase in childhood obesity stands out. Minnesota researchers have demonstrated, that in obese children, oxidative stress, endothelial dysfunction, and elevated cholesterol levels are common metabolic risks [32]. They also have shown, that just eight seeks of moderate exercise, improves fitness, normalizes endothelial function and lowers LDL-cholesterol. Other than the metabolic risks we have discussed before, oxidative stress and inflammation, seems to be relatively early risk markers. In the process of converting food into chemical energy (normal metabolism), mitochondria produce a chemical called superoxide (SO), which has a critical role in cells, but is toxic in excess. Because of this toxicity, mitochondria also produce an enzyme called superoxide dismutase (SOD) to convert superoxide to a less toxic form. Researchers at the Salk Institute have found out for the first time, that a brief exposure to short term stress can be beneficial, by prompting cell to trigger sustained production of antioxidants, molecules that help get rid of toxic cellular buildup of metabolites, related to normal metabolism [33]. 
A new study reported from Doug Seals and Chris Martens of Department of Integrative Physiology, Colorado University, Boulder, shows that six weeks of $500 \mathrm{mg}$ twice daily of nicotinamide riboside (NR) chloride (NIAGEN) boosts the level of nicotinamide adenine nucleotide (NAD+) and reverses the physiological signs of aging and kick starts the same key chemical pathways responsible for its health benefits, including the activation of enzymes called sirtuins, which are largely credited for the beneficial effects of calorie restriction $[34,35]$. Just like inflammation plays a role, in some of the natural physiological processes, oxidative stress, and reactive oxygen species (ROS), also play an important role in cell signaling and homeostasis. According to the researchers on this topic, oxidative stress has a central role in the pathogenesis of atherosclerosis [36-38]. In fact, increased generation of ROS in the vessel wall, and a reduction in the production of vasodilator like nitric oxide (NO), will lead to an imbalance in the ratios of vaso constrictor to vasodilators, and thus initiate endothelial dysfunction, which we consider is the earliest sign of vascular disease. In addition, they can also trigger several redox-sensitive transcriptional pathways, shifting cellular, molecular response and gene expression profile, towards proatherogenic state.

Thus, they can accelerate formation, accumulation of lipid peroxidation products, and modulate proinflammatory genes. The transcription factor Nrf2 (nuclear factor erythroid-2-reoated factor-2,Nrf-2) for instance, a master regulator of detoxification, antioxidant, anti-inflammatory and other cytoprotective mechanisms, is raised by health promoting factors. This transcription factor activates the transcription of over 500 genes (so called survival genes) in the human genome, most of which have cytoprotective functions. The most healthful diets such as Mediterranean and Okinawa are rich in Nrf2 raising nutrients. Modern diets are deficient in such nutrients. Swedish researchers, involving over 62,000 Danish individuals have demonstrated, that childhood overweight is associated with an increased risk for developing type-2 diabetes in adulthood $[39,40]$. According to a recent report by the Centre for Disease Control (CDC) and Prevention, 50\% of the adults in the USA are prediabetic (84 million).

It is a well-known factor, that the individuals with diabetes have a several fold higher risks for developing vascular disease. High incidence of prediabetes is not unique to the USA, it is a worldwide phenomenon. In view of these observations, it is reasonable to conclude, that worldwide increase in these twin epidemics, obesity and diabetes, will contribute significantly, to the increased vascular disease including CVD. According to all estimates, cardiovascular disease is the number one cause for all-cause mortality worldwide. Having said that, I would like to inform the readers that some recent estimates put the diabetes related deaths to the same level as CVD deaths. Furthermore, studies done in 26 industrialized nations has shown a decline in CVD deaths, whereas the same study reports an increase in diabetes-related deaths in high income countries [41].

\section{Discussion}

Framingham heart study group, in collaboration with the researchers at the Boston University School of Medicine, have been working on the population-based studies, on risk factors that promote the development of cardiovascular diseases. As they discovered various risk factors, the pharma industry started working on the development of drugs, that would inhibit these observed risk factors. This line of thinking became, a major approach to the management of chronic diseases, with heavy emphasis on the risk factor reduction or reversal. The practice of western medicine became disease centric, rather than preventive. Another factor that contributed to this fiasco, was the separation of School of Public Health from the School of Medicine in major universities, creating a disconnect between the population-based studies and personalized medicine. We strongly believe that understanding the mechanisms, that underlie the development metabolic risks, outweighs the focus on risk factor management.

With this in view, we have discussed some scenarios, and presented our views, on the development of novel approaches for early diagnosis of metabolic risks and a holistic integrated approach to the management of diseases [41-50]. In the ancient system of medicine in India, metabolic alterations are called "doshas", -defects in metabolism. They go one step further and says that all human beings are born with doshas and as such susceptible to risks that are promoted by the inherited doshas [51]. The entire Ayurveda System of therapy as well as Tibetan Medicine is based on balancing these doshas to attain better health. We made a statement early on, in this overview that, all of the known metabolic risks, hypertension, excess weight, obesity, metabolic syndrome, type-2 diabetes, and vascular diseases have reached epidemic proportions worldwide. According a report by the noncommunicable disease task force, chances of meeting the challenge of Millennium Development Goals of stopping, the increase in these metabolic diseases by 2025, to the 2020 level is less than one percent $[52,53]$. Therefore, it is important to develop novel approaches, to diagnose the earliest possible metabolic risks, and initiate appropriate interventional strategies.

In our introductory abstract we mentioned, that, "Modern day biochemistry in the broadest sense, should provide needed insight into the underlying mechanisms of both structure and regulation, that occur at the molecular, cellular, tissue, organ and whole system level". We would like to see a similar approach, in the research as well. We also mentioned about the optimism expressed by Dr Francis Collins, the sixteenth Director of the prestigious National Institutes of Health, USA, about the future of genomics, genes expressions, and gene therapy. As is with other complex diseases, the search for diabetes susceptibility genes, started decades ago. Of the hundreds of candidate genes studied, and more than 30 genome-wide linkage investigations, neither approach has proved particularly successful in identifying diabetes susceptibility loci [54]. However, as we discussed earlier, researchers at NIH have demonstrated a single gene association with BMI in certain populations.

At the molecular and cellular level, a prime example of the early origin of metabolic risk is islets cell dysfunction, resistance to the action of insulin in facilitating glucose metabolism and the ill effects of excess glucose in the blood. Almost three decades ago, 
we demonstrated that in the drug induced hyperglycemia, one can show altered metabolism at various signaling mechanisms including arachidonic acid metabolism (AA) in platelets and vascular tissue. Researchers at the Anderson Cancer Center have shown that sugars promote inflammatory pathways. They also have shown excess 12-HETE a product of AA metabolism in lung metastasis and breast cancers. Our studies in the 80s, demonstrated that islet cell transplantation restored the altered arachidonic acid metabolism in this animal model [55]. In spite of the fact that fasting glucose levels, hemoglobin A1c and post prandial glucose are gold standard parameters used for the management of diabetes and its clinical complications, not much is known about how glucose exerts its toxic effects at the molecular and cellular levels.

Bulk of the work on the role of glucose in metabolic risks, is related to its ability to glycate certain proteins, including advance glycated end (AGE) products. Recent studies suggest a role for glucose-DNA alterations leading to the development of cancer. There seems to be an association between excess glucose (diabetes) and cancer. However, what is not very clear is why are women with diabetes more prone to get cancer than men? There is lots of opportunities for research and exploration, at the molecular and cellular level, as well as detection and management of early biomarkers that promote metabolic risks. Bilateral studies between the Children's National Medical Hospital and Diabetes Group at the KEM Hospital, Pune, India has explored the role of micro RNAs in the fetal origin of metabolic risks associated with obesity. In view of the importance of this seminal finding, which could a be game changer, in our understanding of the pathology related to obesity related diseases, prestigious National Institute of Health, USA, has funded these bilateral studies.

Studies on this topic in India, has also demonstrated the role of intrauterine micronutrient deficiency, in the pathogenesis of fetal origin of adult diseases [20]. Studies at the University of Texas, USA, has reported that micronutrient deficient diet may be responsible for the excess diabetes in the South Asians living in the USA [56]. There seems to be a great window of opportunity for development of nutritional therapies. Metabolic alterations at the tissue level are very much evident, when we explore the South Asian phenotype, which is predominantly characterized by central abdominal obesity (Visceral obesity). Visceral fat is supposed to be a major cause for the low-grade inflammation in this population.

The results of epidemiological, as well as physiological studies, have demonstrated a strong association with excess abdominal adipose tissue, and the presence of metabolic risks for coronary artery disease, including insulin resistance, impaired glucose tolerance, dyslipidemia, and increased circulatory inflammatory proteins [57]. We can demonstrate system level variation in ethnicity-based metabolic risks. Prime example will be, excess metabolic risks such as diabetes and heart disease in South Asians living abroad. In a short overview like this, it is not possible to cover all aspects of biomedical education and research, readers are urged to refer to original article, reviews and monographs. We have just discussed few examples, and presented our views on this topic, to initiate some constructive dialogue and "tweak" the curiosity of the readers [58].

\section{Conclusion}

In view of the tremendous progress made in the various subspecialties of biomedicine and technology, expectations are high, as far as finding cures for noncommunicable diseases, like cancer, obesity, type- 2 diabetes, and vascular diseases. However, progress made in the areas like genomics, bioengineering, regenerative medicine, although are significant have not helped in reducing, reversing or preventing these diseases. Currently, no country has reduced the incidence of these diseases. In major academic institutions, there is considerable interest in revising curricula to meet the ever-increasing need for collective knowledge to offer better healthcare. There is significant improvement in the care of the individual in these specialized centers of excellence. Having said that, we and others note that there is lack of excitement, dedication and passion in the preventive care at the population level. The question then is, who will lead the preventive medicine? There are several stake holders and unless there is a serious commitment from all the stakeholders, reducing any of the metabolic diseases is a far cry.

Biomedical education is continuously evolving. For a long time, the basic sciences taught at the premier medical schools were, bacteriology, biochemistry, hematology and histology. Modern day biomedicine in the broadest sense, should provide needed insight into the underlying mechanisms of both structure and regulation, that occur at the molecular, cellular, tissue, organ and whole system level. We have discussed the changes, that are taking place at several medical institutions in their curricula. Like in any other specialized fields, the recent progress made in multiple disciplines is so rapid, it is hard to catch up, with all the emerging technologies, and integrate them in any curricula. Future of medicine, especially the precision and personal medicine, lies in clinicians gaining much more detailed information about the patient, the underlying causes of the disease, the knowledge of the emerging technologies, and their applications, to deliver personalized or precision treatment, with a better outcome.

In view of the fact, that my area of expertise is cardiovascular research, we have briefly discussed the progress made in this field, with special emphasis for the diagnosis of early metabolic risks, risk reduction, and prevention of metabolic diseases. What we have tried to emphasize, is that reduction or reversal of metabolic risks, is more important than management of observed risks. In spite of the extraordinary progress made in the biomedical sciences, no country has reduced or reversed, the increase in the incidence of metabolic diseases. In our opinion, the focus should be on understanding the mechanisms, that underlie the development of metabolic risks at molecular, cellular, tissue, and organ level and develop appropriate interventions to reduce, reverse or prevent the development of metabolic diseases. Specific purpose of writing this overview, was to make some provocative suggestions, provide some novel examples, and initiate a constructive dialogue related to the biomedical education as well as research. We have not made any attempt to discuss complexities related to the overall medical education or research. We have just used this opportunity, to express our views from personal experience gained, as a faculty in one of the major Academic Health Center. 


\section{References}

1. Rao GHR (2018) Manual of Platelet Morphology, Physiology and Pharmacology. JP Medical Publishers.

2. Campbell PN (1975) Teaching biochemistry to medical students Biochemical Education 3(1): 3-7.

3. Collins FS, Gottlieb S (2018) The next phase of human gene-therapy oversight. N Engl J Med.

4. Rae M (2011) Doris Taylor: recellularized human hearts may be weeks away. Research Blog April.

5. Ott HC, Matthiesen TS, Goh SK, Black LD, Kren SM, et al. (2008) Perfusiondecellularized matrix using nature's platform to engineer a bioartificial heart. Nat Med 14(2): 213-221.

6. Bajwa M (2014) Emerging $21^{\text {st }}$ century medical technologies. Pak J Med. Sci 30(3): 649-655.

7. Russel JH, Stahl PD, Stephenson J, Whelan A (2004) Biomedical education in the 21st century. Mo. Med. 101(5): 484-486.

8. Welling R, Grannan K, Boberg J, Pierce-Boggs K, Engel A (2009) Graduate medical education as the driver for quality and patient safety: a national initiative of independent academic centers. J Surg Educ 66(6): 336-339.

9. Fred H (2012) Medical education on the brink: 62 years of front-line observations and opinions. Tex Heart Inst J 39(3): 322-329.

10. Frenkel J, Chen HC, Ten Cate O (2016) Patient-centered teaching in a technology-dominated era. South Med J 109(12): 743-746.

11. Moses H, Martin JB (2011) Biomedical research and health advances. N Engl J Med 364: 567-571.

12. Russell JH, Stahl PD, Stephenson J, Whelan A (2004) Biomedica Education in the $21^{\text {st }}$ century. Mo Med 101(5): 484-486.

13. Roth GA, Johnson C, Abajobir A, Abd-Allah F, Abera SF, et al. (2017) Global, Regional, and National burden of cardiovascular diseases for 10 causes, 1990-2015. J Am Coll Cardiol 70(1): 1-25.

14. Finucane MM, Stevens GA, Cowan M, Danaei G, Lin JK, et al. (2011) National, and global trends in body mass index since 1980: Systematic analysis of health examination surveys and epidemiological studies with 960 country-years and 9.1 million participants. Lancet 377(9765): $557-$ 567.

15. Daniel G, Finucane KK, Singh GM, Paciorek CJ, Cowan MJ, et al. (2011) National, and global trends in systolic blood pressure since 1980 systematic analysis of health examination surveys and epidemiological studies with 786 country-years and 5.4 million participants. Lancet 377 (9765): 568-577.

16. Farzadfar F, Finucane MM, Daniel G, Pelizzari PM, Cowan MJ, et al. (2011) National, regional and global trends in serum total cholesterol since 1980: a systematic analysis of health examination surveys and epidemiological studies with 321 country-years and 3.0 million participants. Lancet 377(9765): 578-586.

17. (2016) NCD Risk Factor Collaboration (NCD-Ris-C): Worldwide trends in diabetes since 1980: a pooled analysis of 751 population -based studies with 4.4 million participants. Lancet 387(10027): 1513-1530.

18. Cohn JN, Hoke L, Whitwam W, Sommers PA, Taylor AL, et al. (2003) Screening for early detection of cardiovascular disease in asymptomatic individuals. Am Heart J 146(4): 679-685.

19. Fernandez-Friera L, Fuster V, Lopez-Melgar B, Oliva B, García-Ruiz JM, et al. (2017) Normal LDL-cholesterol levels are associated with subclinical atherosclerosis in the absence of risk factors. J Am Coll Cardiol 70(24): 2979-2991.

20. Rao GH, Bharathi M (2016) Mother and child: First step for the prevention of cardiometabolic diseases. J Cardiol (Photon Journal) Photon 109: 179-186.

21. Krishna M, Kalyanaraman K, Veena SR, Krishnaveni GV, Karat SC, et al. (2015) Cohort profile: The 1934-66 Mysore birth records cohort in South India. Int J Epidemiol 44: 1833-1841.
22. Xiao X, Zhang Z, Li W, Feng K, Sun Q, et al. (2010) Low birth weight is associated with components of the metabolic syndrome. Metab 59(9): 1282-1286.

23. Barker DJ (2004) The developmental origins of adult disease. J Am Coll Nutr 23(6): 588S-595S.

24. Barker DJ: The developmental origin of insulin resistance Horm Res 64 (3): 207

25. Kulkarni ML, Mythri HP, Kulkarni AM (2009) “Thinfat" phenotype in newborns. Ind J Pediatr 76(4): 369-373.

26. Garofano A, Czernichow P, Breant B (1997) In utero undernutrition impairs bet-cell development. Diabetologia 40(10): 1231-1234.

27. Calkins K, Devaskar SU (2011) Fetal Origin of adult disease. Curr Probl Pediatr Adoles Health Care 41(6): 158-176.

28. Skogen JC, Overland S (2012) The fetal origins of adult disease: a narrative review of the epidemiological literature. JRSM Short Rep 3(8): 59-65.

29. Ferrante SC, Nadler EP, Pillai D, Hubal MJ, Wang Z, et al. (2015) Adipocyte-derived exosomal miRNAs: a novel mechanism for obesityrelated disease. Ped Res 77(3): 447-454.

30. Wang TJ, Larson MG, Ramachandran SV, Cheng S, Rhee EP, et al. (2011) Metabolite profiles and the risk of developing diabetes. Nat Med 176(4): 448-453.

31. Wurtz P, Soininen P, Kangas A, Rönnemaa T, Lehtimäki T, et al. (2013) Branched chain aromatic amino acids are predictor of insulin resistance in young adults. Daib Care 36(3): 648-655.

32. Kelly A, Wetzateon RJ, Kaiser DR, Steinberger J, Bank AJ, et al. (2004) Inflammation, insulin resistance and endothelial dysfunction in overweight children and adolescents. The J Pediatr 145(6): 731-736.

33. Cox CS, McKay SE, Holmbeck MA, Brooke E Christian, Andrew C Scortea, et al. (2018) Mitohormesis in mice via sustained basal activation of mitochondrial and antioxidant signaling. Cell Metab.

34. Rossman MJ, Santos-Parker JR, Steward CAC, Bispham NZ, Cuevas LM, et al. (2018) Chronic supplementation with a mitochondrial antioxidant (MitoQ) improves vascular function in healthy adults Hypertension 71(6): 1056-1063.

35. Martens CR, Denman BA, Mazzo RM, Michael LA, Reisdorph N, et al (2018) Chronic nicotinamide riboside supplementation is well tolerated and elevates NAD+ in healthy middle-aged and older adults. Nature comm. 1286.

36. Boggard HJ, Natarajan R, Henderson R, Carlin S Long, Donatas Kraskauskas, et al. (2009) Chronic pulmonary artery pressure elevation is insufficient to explain right heart failure. Circ 120: 1951-1960.

37. Hybertson BM, Gao B, Bose SK McCord JM (2011) Oxidative stress in health and disease: The therapeutic potential of $\mathrm{Nrf}_{2}$ activation. Mol Aspects of Med 32: 234-246.

38. Joe M McCord, Fridovich I (1978) The biology and pathology of oxygen radicals. Ann Int Med 89(1): 122-127.

39. Bjerregaard LG, Jensen BW, Angquist L et al. (2018) Change in overweight from childhood to early adulthood and risk for type-2 diabetes. New Engl J Med 378: 1302-1312.

40. Ali MK, Bullard KM, Sayadah S, Imperatore G, Gregg EW (2018) Cardiovascular and renal burdens of prediabetes in the USA: analysis of data from serial cross-sectional surveys, 1988-2014 Lancet Diabetes Endocrinol 6(5): 392-403.

41. Di Cesare M, Bennett JE, Best N, Stevens GA, Danaei G, et al. (2013) The contributions of risk factor trends to cardiometabolic morality declines in 26 industrialized countries. Int J Epidemiol 42(3): 838-848.

42. Rao, GHR, Nagendra HR (2013) Holistic approach for the management of heart disease and type-2 diabetes. J Preven Cardiol 2(1): 231-238.

43. Rao GHR (2018) Excess weight, obesity, diabetes (type2), and clinical complications. Arch Diab \& Obesity 1(1). 
44. Rao GHR (2018) Prevention or reversal of cardiometabolic diseases. J Clin Prevent Cardiol 7(1): 22-28.

45. Rao GHR (2018) Importance of studying disease and disease process. Editorial Developments in Clin Med Pathol Crimson Publ 1(1).

46. GHR (2018) Modern Day Cardiology: Expectations and Limitations. J Cardiol 2(2): 000116.

47. Rao GHR (2018) Cellular and Molecular Approaches to Therapy. Clin Med Pathology 1(3).

48. Rao GHR (2018) Integration of novel emerging technologies for the management of type-2 diabetes. Arch Diab \& Obesity 1(1).

49. Rao GHR (2018) Risk scores for acute vascular events: Expectations and limitations. J Cardiol 2(3).

50. Rao GHR (2018) Reduction reversal or prevention of type-2 diabetes mellitus. Editorial. Arch Endocrinology and Diabetes Care 1(1).

51. Rao GHR (2018) Diagnosis of early risks, management of risks, and reduction of vascular diseases. J Clin Card \& Diagnostics 1(1): 1-11.

52. Lakhotia SC (2014) Translating Ayurveda's Dosha-Prakriti into objective parameters. J Ayurveda Integr Med 5(3): 167-175.

ISSN: 2574-1241

DOI: 10.26717/BJSTR.2018.09.001787

Gundu HR Rao. Biomed J Sci \& Tech Res

(C) (i) This work is licensed under Creative

Submission Link: https://biomedres.us/submit-manuscript.php
53. (2017) NCD Risk Factor Collaboration (NCD-Ris-C): Worldwide trends in bod-mass index, underweight, overweight, and obesity from 19752016: a pooled analysis of 2416 population-based measurement studies in 128.9 million children, adolescent, and adults. Lancet 390 (10113): 2627-2642.

54. (2016) NCD Risk Factor Collaboration (NCD-Ris-C): Worldwide trends in diabetes since 1980: a pooled analysis of 751 population-based studies with 4.4 million participants. Lancet. 387 (10027): 1513-1530.

55. McCarthy MI, Zeggini E (2009) Genome-wide association studies in type-2 diabetes. Curr Diab Rep 9(2): 164-171.

56. Gerrard JM, Stuart MJ, Rao GHR, Steffes MW, Mauer SM, et al. (1980) Alterations in the balance of prostaglandin and thromboxane synthesis in diabetic rats. J Lab Clin Med 95(6): 950-958.

57. Shah M, Vasandani C, Adams-Huet B, Garg A (2018) Comparison of nutrient intakes in South Asians with type-2 diabetes mellitus and controls living in the United States. Diab Res Clin Pract 138: 47-56.

58. Klein Samuel (2010) Is visceral fat responsible for the metabolic abnormalities associated with obesity? Diab Care 33(7): 1693-1694.

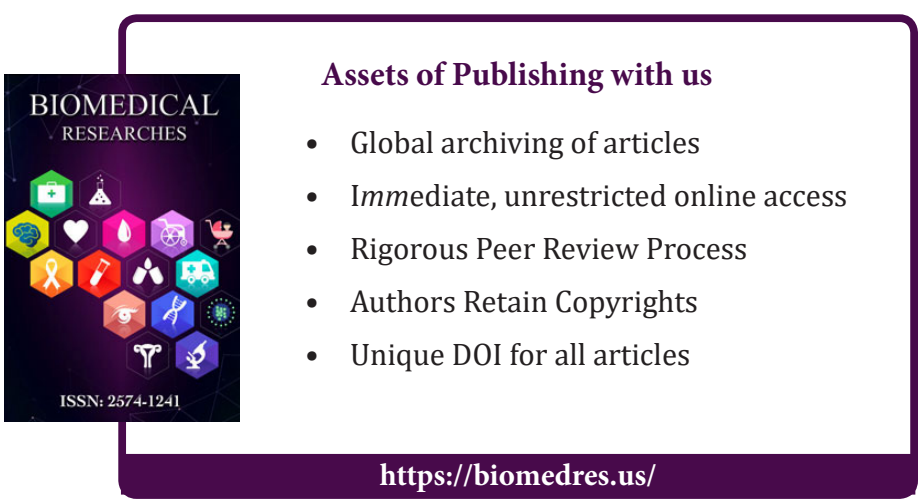

